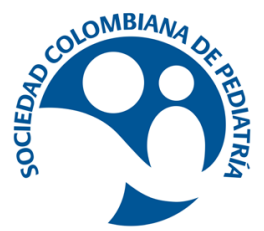

\title{
Original
}

\section{Vivencia de madres de prematuros con oxígeno en un programa madre canguro de Bogotá, Colombia*}

\author{
Sonia E. Guevara-Suta ${ }^{a}$, Martha O. Ospina-Rubiano ${ }^{a}$, Héctor F. Restrepo-Guerrero ${ }^{b}$ \\ a Magíster en Salud Pública y Desarrollo Social. Docente Programa de Terapia Respiratoria, Facultad de Ciencias de la Salud y el Deporte, \\ Fundación Universitaria del Área Andina, Bogotá, Colombia \\ b Médico Veterinario Zootecnista. PhD Gestión de proyectos. Msc Epidemiología. Fundación Universitaria de Ciencias de la Salud, Bogotá, \\ Colombia.
}

INFORMACIÓN DEL ARTÍCULO

\section{Historia del artículo:}

Recibido el 26 de agosto 2020

Aceptado el 20 de noviembre de 2020

\section{Palabras clave:}

Terapia por inhalación de oxígeno recién nacido prematuro método madre-canguro.

*Aire Libre Research Group, Respiratory Therapy Program, School of Health and Sport Sciences, Fundación Universitaria del Área Andina, Bogotá, Colombia

\section{R E S U M E N}

Introducción: El nacimiento prematuro es considerado un problema de salud pública y es el responsable de una alta morbimortalidad neonatal. Un gran porcentaje de prematuros requieren de oxígeno y de ventilación mecánica para suplir sus necesidades fisiológicas y cerca de un $40 \%$ de ellos son dados de alta de las unidades neonatales y dirigidos a los programas madre canguro. Objetivo: Describir las vivencias de madres de bebés con oxígeno admitidos en un programa madre canguro, desde su contexto cultural. Métodos: Estudio cualitativo, etnográfico. Se aplicó una entrevista semiestructurada a diez y nueve madres de bebés con oxígeno, con edades entre 16-38 años. Resultados: Cuatro categorías: identificación de signos de alarma respiratorios y cuidados de equipos en casa; adaptación frente al manejo con oxígeno; relación con escenario cultural y asistencia a controles de oximetría, las cuales surgieron del proceso de análisis de las entrevistas. Conclusiones: Se debe garantizar a los padres la accesibilidad a los servicios de salud, con el fin de dar continuidad a los procesos de seguimiento que requieren los niños prematuros en los programas madre canguro ambulatorio. La investigación permite evidenciar que el Programa Ruta de la Salud que les ofrecen a los padres no es suficiente y requiere de una mayor cobertura. Se debe generar una estrategia que permita realizar un seguimiento y acompañamiento a través de la teleorientación, con el fin de abordar inquietudes que puedan surgir en casa y disminuir en ellos los niveles de ansiedad y estrés.

\footnotetext{
${ }^{*}$ Autor para correspondencia. Sonia Esperanza Guevara Suta Correo electrónico: sguevara@areandina.edu.co
}

Como Citar: Guevara-Suta SE, Ospina-Rubiano MO, Restrepo-Guerrero HF. Vivencia de madres de prematuros con oxígeno en un programa madre canguro de Bogotá, Colombia Pediatr. 2020;53(2):56-63. 


\title{
Experience of mothers of premature babies with oxygen in a kangaroo mother program in Bogota, Colombia*
}

\author{
A B S T R A C T
}

Keywords:

Oxygen Inhalation Therapy

infant, Premature

kangaroo-Mother Care Method
Introduction: Preterm birth is considered a public health problem and is responsible for high neonatal morbidity and mortality. A large percentage of premature infants require oxygen and mechanical ventilation to meet their physiological needs, and about $40 \%$ of them are discharged from neonatal units to the Kangaroo Mother Program. Objective: To describe the experiences of mothers of babies with oxygen admitted to a kangaroo mother program, from their cultural context. Methods: A qualitative, ethnographic study, a semi-structured interview was applied to nineteen mothers of babies with oxygen, aged between 16-38 years. Results: Four categories: identification of respiratory alarm signs and home care equipment; adaptation to handling with oxygen; relationship with cultural setting and attendance at oximetry controls, which arose from analyzing the interviews. Conclusions: Accessibility to health services must be guaranteed to parents; to give continuity to the follow-up processes required by premature infants in the Ambulatory Kangaroo Mother Programs, the research shows that the Health Route Program offered to parents is not enough and needs more coverage. A strategy must be generated that allows monitoring and accompaniment through teleorientation to address concerns that may arise at home and reduce anxiety and stress levels in them.

\section{Introducción}

La prematuridad a nivel mundial se constituye en una causa importante de mortalidad y morbilidad neonatal. La Organización Mundial de la Salud en el año 2018 estimó que uno de cada diez niños nace prematuramente (nacido vivo antes de las 37 semanas de gestación) y cerca de un millón de ellos mueren por complicaciones. En América Latina y el Caribe el país que más registró nacimientos prematuros fue Costa Rica (13.6\%), seguido de El Salvador (12.8\%). En Colombia alrededor del $12 \%$ de los nacimientos son prematuros y su mortalidad está asociada al desarrollo de patologías como displasia broncopulmonar (1).

En la actualidad, la reducción de la morbimortalidad neonatal ha sido posible gracias al seguimiento de controles prenatales, a un acceso sin restricciones de los servicios de salud y a los avances médicos y tecnológicos para el cuidado prenatal. En los nacimientos prematuros la secuela respiratoria más prevalente sigue siendo la displasia broncopulmonar (DBP) y una de las que más condiciona su pronóstico (2). La displasia broncopulmonar continúa representando una causa significativa de morbilidad entre los sobrevivientes de prematuridad en las unidades neonatales (3). Esta patología se asocia a causas como uso prolongado de oxígeno, ocasionando una mayor incidencia de problemas respiratorios y cardiovasculares en los dos primeros años de vida (4).

El cuidado de estos pacientes prematuros sigue siendo costoso para los sistemas de salud, por ello, el método madre canguro (MMC) se convierte en una alternativa costo-efectiva, en razón, a que se basa en un modelo humanizado y seguro para la madre y el niño, cuyas premisas fundamentales son el calor, amor y la lactancia materna, fundamentales para la sobrevida porque aportan la mejor nutrición, activan el sistema inmune, favorecen el contacto piel con piel, el apego y el afecto, así como reducen los períodos de estancia hospitalaria, el uso de incubadoras y la administración de medicamentos.

Para el seguimiento de los pacientes neonatales que egresan de las unidades neonatales existe también un programa de seguimiento de alto riesgo centrado en la atención de pacientes con encefalopatía hipóxico-isquémica, cardiopatías congénitas y malformaciones complejas (5).

Durante la hospitalización del paciente prematuro el talento humano en salud es el encargado de los cuidados generales, participa en su recuperación y está en continuo contacto con los padres, de ahí la importancia que el personal de la unidad neonatal trabaje con un método inductivo, enfocado en las personas y no únicamente en la enfermedad (6).

Una vez el prematuro se encuentra estable hemodinámicamente, se debe educar a los padres para que se conviertan en los cuidadores primarios en el hogar, con la transferencia gradual de la responsabilidad del cuidado y las habilidades para ello. Las capacitaciones se deben orientar en las técnicas de posición canguro, lactancia materna exclusiva hasta los seis meses, dado que es un alimento importante para su crecimiento y desarrollo, y estrategias de apoyo en el servicio de salud con las que cuenta la madre para el acompañamiento y mantenimiento de la práctica, si el paciente requiere de oxígeno se debe fortalecer los cuidados de los equipos de oxigenoterapia en casa.

Al egreso hospitalario se le debe asegurar al paciente el ingreso al programa madre canguro (PMC), con seguimiento ambulatorio estricto al menos durante los dos primeros años de vida. Los niños con oxígeno requieren acompañamiento continúo por profesionales entrenados, para que inicien un retiro progresivo del mismo. El presente artículo describe las vivencias de madres de bebés con oxígeno admitidos en un programa madre canguro de Bogotá, Colombia, desde su contexto cultural. 


\section{Métodos}

Tipo de estudio. Estudio cualitativo con abordaje etnográfico, donde se describen las vivencias de madres de bebés con oxígeno que asisten al programa madre canguro de la Subred Sur de Bogotá, Colombia. El núcleo de enfoque de la investigación se centró en las vivencias de las madres de los bebés con oxígeno. Para investigar en prematuros se exige tener presente la situación y el tema de estudio, valorar y discutir todos los aspectos que forman parte de su historia, entorno y de la realidad donde está inmersa; sus valores, tradiciones, creencias y diferentes modos de vivir relacionados con su construcción social (7).

\section{Muestra.}

Conformada por diez y nueve madres de bebés que requirieron oxígeno. Las edades de las madres estuvieron entre 16 y 38 años. Se realizaron entrevistas semiestructuradas de observación directa.

\section{Criterios de inclusión.}

Ser madres de prematuros con requerimiento de oxígeno que asistan al PMC de la Subred Sur y ser madres de pacientes prematuros sin malformaciones congénitas. Una vez seleccionados los pacientes que cumplieron con los criterios de inclusión, se contactó a las madres para explicarle las características del estudio e invitarlas a participar en el trabajo de investigación, quienes aceptaron participar voluntariamente y firmaron el consentimiento informado.

\section{Recolección de la información.}

La información suministrada por las madres fue recolectada por las investigadoras. Se realizó una entrevista a las madres en donde se grabó cada relato en una grabadora digital, abarcando un tiempo de entrevista de 25 a 30 minutos. Las entrevistas fueron transcritas por las investigadoras. Cada entrevista fue orientada con una guía temática y también se permitió que las madres hablaran libre y espontáneamente. Se destacó la disponibilidad de ellas para compartir sus vivencias.

\section{Interpretación de la información.}

El análisis de la información suministrada se realizó en dos momentos: uno durante la recolección de la información, y el otro después de verificar la transcripción literal de cada entrevista. Las investigadoras analizaron el texto para iniciar la comprensión de las experiencias de las participantes y para identificar aspectos que debían profundizarse. Al finalizar la recolección de la información se procedió con el análisis de cada entrevista. Para esto se leyó el texto de estas y se escribió la experiencia compartida por cada participante, la cual fue revisada y registrada. Todas las historias se escribieron y se clasificaron en cuatro cuadros de Excel $\circledast$, se buscaron similitudes, diferencias y patrones en cada historia narrada por las madres lo que permitió identificar los temas y subtemas para describir sus vivencias.

Para la protección de la identidad de las participantes, se decidió enumerar a las madres de manera consecutiva desde el número 1 al 19, lo que corresponde al total de las madres entrevistadas. Para el análisis de la información se organizaron tablas en Excel que correspondieron a la información recolectada, así: tabla 1 variables sociodemográficas, tabla 2 variables fisiopatológicas maternas, tabla 3 variables de nacimiento y tabla 4 categorías analíticas. Para velar por el rigor metodológico durante el estudio se tuvieron en cuenta los criterios de credibilidad o validez interna y auditabilidad o confirmabilidad de Guba y Lincoln (8).

\section{Tabla 1. Variables sociodemográficas}

Edad de las madres (años) 16 - 38

\begin{tabular}{|c|c|c|c|}
\hline \multirow{2}{*}{ Escolaridad } & Bachillerato Completo & Bachillerato Incompleto & Especialización \\
\hline & $63.1 \%$ & $31.6 \%$ & $5.2 \%$ \\
\hline \multirow{2}{*}{ Ocupación } & Ama De Casa & Vendedora & Ingeniería \\
\hline & $78.9 \%$ & $15.7 \%$ & $5.2 \%$ \\
\hline \multirow{2}{*}{ Nacionalidad } & Colombiana & \multicolumn{2}{|c|}{ Venezolana } \\
\hline & $68.5 \%$ & \multicolumn{2}{|c|}{$31.5 \%$} \\
\hline \multirow{2}{*}{ Convivencia } & Esposo e hijos & Hijos & Padres \\
\hline & $90 \%$ & $5.2 \%$ & $5.2 \%$ \\
\hline \multirow{2}{*}{ Número de hijos } & 2 & 3 & 6 \\
\hline & $31.6 \%$ & $24.3 \%$ & $5.2 \%$ \\
\hline $\begin{array}{l}\text { Número de personas que habitan la } \\
\text { vivienda }\end{array}$ & Mínimo: 4 & \multicolumn{2}{|c|}{ Máximo: 40} \\
\hline \multirow{2}{*}{ Número de habitaciones donde habita } & 2 & 3 & 6 \\
\hline & $63.3 \%$ & $21 \%$ & $5.2 \%$ \\
\hline \multirow{2}{*}{ Estrato socioeconómico } & 1 & 2 & 3 \\
\hline & $42.2 \%$ & $47.4 \%$ & $10.4 \%$ \\
\hline Servicios públicos (agua, luz, gas) & \multicolumn{3}{|c|}{$100 \%$} \\
\hline
\end{tabular}


Tabla 2. Variables Fisiopatológicas Maternas

\begin{tabular}{lll} 
Control Prenatal & Si: $100 \%$ & \\
\hline Embarazo Planeado & Si: $42 \%$ & No: $58 \%$ \\
\hline & Infección de vías urinarias & Pre eclampsia \\
\cline { 2 - 3 } $\begin{array}{l}\text { Antecedentes del } \\
\text { embarazo }\end{array}$ & $10.5 \%$ & $15.4 \%$ \\
\cline { 2 - 3 } & Prematurez & Sin Antecedentes \\
\cline { 2 - 3 } & $10.5 \%$ & $63.2 \%$ \\
\hline
\end{tabular}

\begin{tabular}{lll} 
Tabla 3. Variables & De Nacimiento \\
\hline \multirow{3}{*}{ Semanas EG* } & Menores de 32 semanas de EG & $36.8 \%$ \\
\cline { 2 - 3 } & De 33 a 36 semanas de EG & $42 \%$ \\
\cline { 2 - 3 } & Mayores de 37 semanas de EG & $21 \%$ \\
\hline \multirow{3}{*}{ Peso (gramos) } & Menores de 1000 gramos. & $10.52 \%$ \\
\cline { 2 - 3 } & De 1000 - 2000 gramos. & $47.36 \%$ \\
\cline { 2 - 3 } & Mayores de 2000 gramos. & $42.10 \%$ \\
\hline \multirow{2}{*}{ Sexo } & Femenino & $73.9 \%$ \\
\cline { 2 - 3 } & Masculino & $26.5 \%$ \\
\hline \multirow{3}{*}{ Tipo De Embarazo } & Embarazo gemelar y/o mellizos & $10.4 \%$ \\
\cline { 2 - 3 } & Embarazo múltiple (Trillizas) & $5.2 \%$ \\
\cline { 2 - 3 } & Embarazo Único & $84.4 \%$ \\
\hline \multirow{2}{*}{ Vía Del Parto } & Cesárea & $68.42 \%$ \\
\cline { 2 - 3 } & Vaginal & $31.57 \%$ \\
\hline EG: Edad gestacional & \\
\hline
\end{tabular}

Para evitar imprimir un sesgo personal a la descripción de las vivencias de las madres se realizó la inmersión en la información obtenida a través de la entrevista e historias que contaron las madres sobre el cuidado del bebé con oxígeno en casa, buscando de manera consciente ceñirse a los datos registrados. La transferibilidad del estudio investigativo fue determinada por los lectores de este artículo al juzgar y verificar si los resultados describen las vivencias de las madres que afrontan el egreso de la unidad neonatal de su hijo con oxígeno.

\section{Aspectos éticos.}

Para el desarrollo del presente estudio se tuvo en cuenta la resolución 8430 de octubre 4 de 1993, en la que se establecen las normas científicas, técnicas y administrativas para la investigación en salud en Colombia. Se respetaron los principios éticos contemplados en la declaración de Helsinki (9). El presente estudio es de riesgo mínimo, se tratarán temas sensibles en población vulnerable. El estudio fue aprobado por el comité de ética de la investigación de la Subred Sur.

\section{Resultados}

Al realizar un análisis de las entrevistas, se encontró que la edad de las madres oscilo entre 16 y 38 años. Relacionado con la escolaridad de las participantes, el $63.1 \%$ de las madres tienen bachillerato completo, el $31.6 \%$ bachillerato incompleto y el $5.2 \%$ corresponde a una madre con especialización. Frente a la ocupación de las madres el 78.9 \% se dedican al hogar. La nacionalidad de las madres en un $68.5 \%$ son colombianas y un $31.5 \%$ son de nacionalidad venezolana. El $89.6 \%$ de las madres conviven con el esposo. Relacionado con la gravidez, se encontró que para $31.6 \%$ de las mujeres era el primer embarazo, el $39 \%$ eran grávidas por segunda vez, y una mamá, es decir, el $5.2 \%$ había estado embarazada seis veces. Referente a las personas que habitan la vivienda se encontró que en el número de personas que conviven es de 4, salvo para una madre de nacionalidad venezolana en donde el número de personas asciende a 40 en una misma casa. El número de habitaciones en la vivienda corresponde a 2 con un $63.3 \%$. Relacionado con el estrato socioeconómico el $47.4 \%$ corresponden a estrato 2, seguido del $42.2 \%$ con el estrato 1 . Todas las participantes cuentan con los servicios públicos básicos.

Referente a las variables del embarazo, el $100 \%$ de las madres se realizaron controles prenatales. El $57.9 \%$ no planearon el embarazo. Según los antecedentes del embarazo el $63.2 \%$ de las madres no presentaron antecedentes durante el embarazo, el $15.4 \%$ presentaron preeclampsia, el $21 \%$ de las madres presentaron infecciones de vías urinarias y prematuridad.

Al analizar las variables de nacimiento el $42.1 \%$ de los nacimientos fueron entre las 33 a 36 semanas de edad 
gestacional (EG) calculada por la prueba de Ballard, el $36.8 \%$ en menores de 32 semanas de EG y el $21 \%$ a mayores de 37 semanas de EG. Relacionado con el peso al nacer el $47.4 \%$ estuvo entre 1000 a 2000 gramos. El $42.1 \%$ con peso mayor a 2000 gramos y el $10.5 \%$ a menores de 1000 gramos. El $74 \%$ de los nacimientos corresponden a sexo femenino; según el tipo de embarazo $84.4 \%$ fue embarazo único. Se resalta que de las entrevistadas cursó con embarazo gemelar, otra con embarazo de mellizos y una mamá con embarazo de trillizas. Relacionado con la vía del parto el 68.4 \% fue por vía abdominal. Ver tabla 1 de variables sociodemográficas, fisiopatológicas y de nacimiento.

\section{Categorías analíticas}

Las madres hablaron sobre las vivencias desde el egreso hospitalario y la adaptación del primer día en casa con su bebé. Se evidenciaron cuatro categorías: signos de alarma respiratorios y cuidados de equipos de oxigenoterapia, adaptación de las madres frente al manejo del oxígeno, relación con el escenario cultural y asistencia a controles de oximetría. Para mayor comprensión se reflexiona sobre los hallazgos encontrados. Ver tabla 4 de categorías analíticas.

\section{Signos de alarma respiratorios y cuidado de equipos de oxigenoterapia.}

Las participantes del estudio manifestaron que durante la hospitalización recibieron capacitaciones, entrenamiento y acompañamiento por parte de los terapeutas respiratorios sobre signos de alarma respiratorios, el manejo del oxígeno y los cuidados que se deben tener en casa. A continuación, se relatan algunos testimonios de las madres durante la entrevista. Una madre manifestó que le explicaron que: «por el oxígeno pueden perder la vista por durar mucho tiempo, la visión se disminuye por el oxígeno».

Otra madre índica: «la terapeuta respiratoria me explico los cuidados con las balas de oxígeno, que no se caiga, que no esté cerca del calor y me explico los signos de alarma respiratorio".

Otro testimonio fue: «todavía no se dejar quitar el oxígeno, no satura bien cuando come, está muy dormida». Otra madre índica: «nos enseñaron a identificar los signos de alarma y la dificultad respiratoria, nos enseñaron unas terapias y lavados nasales». Una madre manifestó: «me explicaron los signos de alarma respiratorios, el manejo del oxígeno y los cuidados que se deben tener en casa con las balas de oxígeno». Otro testimonio indico que: «nos capacitaron para el manejo del oxígeno en casa y los cuidados de las balas».

\section{Adaptación de las madres frente al manejo del oxígeno}

Las madres después de recibir capacitaciones sobre los cuidados del oxígeno y el entrenamiento en plan canguro tienen una meta clara y es el cuidado de su bebe casa, aunque saben que este proceso no es fácil, reconocen que su bebé estará mejor en casa que en el hospital. Las participantes expresaron sentimientos en el proceso de adaptación, como dudas, temores e inseguridades especialmente durante la primera noche. Un aspecto referido es el compromiso y responsabilidad que sienten de cuidar a su hijo.

Uno de los testimonios de las madres fue: "sentí susto, miedo, no pude dormir por estar pendiente de que la cánula nasal no se saliera y de la comida cada dos horas». Aunque la madre refiere tener experiencia porque su hija mayor fue prematura, resaltó el acompañamiento del esposo en la noche.

Un testimonio expresado fue: «es una lucha diaria, ya que no sólo es darle comida sino estar pendiente del oxígeno, de que no se salga de la nariz, del control del peso y cuando las cosas no salen bien da mucha tristeza»

Una madre indicó: "fue terrible porque tengo otros hijos pequeños de 15, 14, 8, 6, 4 años». Señaló que sintió «susto y pánico de los signos de alarma y que los otros hijos no le fueran a bajar el oxígeno a la bebé». Refirió que el primer día no durmió por miedo al oxígeno y por la comida.

Otro testimonio fue: «sentí preocupación por los signos de alarma respiratorios, sentí emoción, fue algo nuevo", relató que tuvieron que cambiar de vivienda porque donde vivían era demasiado frío y húmedo, nos hizo cambiar la vida para bien..

Una madre expresó que: «la primera y siguientes noches fueron terribles, con nervios, no se pudo dormir por el manejo del oxígeno".

Otro aspecto referido fue: «al papá le dio duro la primera noche por lo del oxígeno, él no durmió, yo dormí de la media noche hasta las 5 de la mañana".

Otro testimonio está relacionado con el sentimiento de nostalgia cuando se le preguntaba durante la hospitalización sobre los cuidados del bebé en casa «uno viene y se aburre porque uno cree que se las sabe todas y cuando uno llega a la casa se da cuenta que no sabe nada y que todo lo que le dijeron en el hospital es por el bien de nuestros niños».

\section{Escenario cultural}

Las madres manifestaron que no vivían cerca del PMC. Dos participantes relataron que para el traslado a los controles lo hacían en taxi por la dificultad que implicaba cargar a su bebé prematuro y al cilindro de oxígeno. Otras madres indicaron que tenían que desplazarse en transporte público por el costo del taxi, el desplazamiento es complicado. A continuación, se describen algunos los testimonios: Una madre relato que: «uso la ruta de la salud en la tarde porque es demorada en la mañana, mi esposo me acompaña en la mañana a coger bus».

Otra madre expresó que: «el transporte es difícil, hay trancones, congestión y me toca caminar hasta llegar a la avenida principal con la bala de oxígeno pequeña y mi otra hija de tres años que me acompaña, casi no pasan taxis, mi hija sale corriendo y es un peligro para ella». Indicó que por este motivo muchas veces llega tarde a la consulta. Otro testimonio fue que: «el desplazamiento es complicado porque me demoro una hora en llegar a los controles, me toca coger bus y siempre vengo sola ya que no hay quien me acompañe, pues mi esposo busca trabajo, mi suegra me ayuda con los gastos económicos y mis otros niños son pequeños»

Una madre indico: «mi esposo me acompaña en la mañana, nos venimos a pie, nos gastamos 20 minutos para llegar a la consulta y me toca devolverme sola porque, no tengo quien me acompañe». Otro de los testimonios es: «me trasporto sola, a mi esposo no le dan permiso en el trabajo, el hospital tiene una ruta y me recogen en un paradero». La madre de las trillizas indico: "siempre vengo acompañada por mi esposo, mi suegra y una enfermera del albergue donde nos quedamos, en un carro del albergue». 


\section{Tabla 4. Categorías Analíticas}

Capacitación de las madres antes del egreso hospitalario:

1. Signos de alarma respiratorios 2. Cuidado de equipos de oxigenoterapia en casa

Adaptación de las madres frente al manejo del oxígeno

Relación con el escenario cultural

Asistencia a controles de oximetría

«Los terapeutas respiratorios nos capacitaron en los signos de alarma respiratorios, el manejo del oxígeno y los cuidados de los cilindros como que no se caiga, que no esté cerca del calor»

«Puede perder la vista por durar tanto tiempo con oxígeno, la visión se disminuye por el oxígeno. Todavía no se dejar quitar el oxígeno, no satura bien cuando come y cuando está dormida»

«Nos enseñaron a identificar los signos de alarma respiratorios y unas terapias de lavados de la nariz».

«Susto, miedo, no se puede dormir por estar pendientes de que la cánula nasal no se saliera de la nariz y de la comida cada 2 horas", aunque la madre refiere tener experiencia porque su hija mayor fue prematura también. El esposo le colabora en la noche.

«Es una lucha diaria, ya que no sólo es darle comida sino estar pendiente del oxígeno, de que no se salga de la nariz, del control del peso y cuando las cosas no salen bien da mucha tristeza»

«Terrible porque tengo otros hijos pequeños. Asustada, con pánico de los signos de alarma y que los otros hijos no le fueran a bajar el oxígeno a mi bebé.

«Preocupación por los signos de alarma respiratorios, emoción, algo nuevo, cambié de vivienda porque donde vivía era muy frío y húmedo, nos cambió la vida para bien»

«Al papá le dio duro la primera noche por lo del oxígeno, él no durmió, yo dormí de la media noche hasta las 5 de la mañana»

«Uno viene y se aburre porque uno cree que se las sabe todas y cuando uno llega a la casa se da cuenta que no sabe nada y que todo lo que le dijeron en el hospital es por el bien de nuestros niños"

«Desplazamiento en taxi por la dificultad que implica cargar a mi bebé prematuro y a la bala portátil de oxígeno»

«Uso la ruta de la salud en la tarde porque es demorada en la mañana, mi esposo me acompaña en la mañana a coger bus»

«El desplazamiento es complicado porque me demoro una hora en llegar a los controles, me toca coger bus y siempre vengo sola ya que no hay quien me acompañe, pues mi esposo busca trabajo, mi suegra me ayuda con los gastos económicos y mis otros niños son pequeños»

«Trasporte difícil, trancones, congestión, tenemos que caminar hasta llegar a la avenida principal con el cilindro y mi niña de 3 años y casi no pasan taxis, mi hija sale corriendo y es un peligro para ella»

«Transporte público, por el costo que genera coger taxi, el desplazamiento es complicado por el cilindro de oxígeno»

«Mi esposo me acompaña en la mañana, nos venimos a pie, nos gastamos 20 minutos para llegar a la consulta y me toca devolverme sola porque, no tengo quien me acompañe»

«Vengo sola, a mi esposo no le dan permiso en el trabajo, el hospital tiene una ruta y me recogen en un paradero"

«Siempre vengo acompañada por mi esposo, mi suegra y una enfermera del albergue donde nos quedamos, en un carro del albergue»

"Asistieron a la consulta al día siguiente de la salida del hospital con sus parejas y acompañantes, pero ellos muchas veces tienen que dejar de ir a trabajar para acompañarlas a los controles, así como dejar de hacer algunas actividades de su vida cotidiana"

"Acudieron al control del oxígeno como fue indicado antes de la salida de la unidad neonatal y han asistido a todos los controles programados de oxígeno»

«No ha asistido a todos los controles de oxígeno porque tiene otros niños pequeños y no tiene quién se los cuide»

\section{Asistencia a controles de oximetría}

La experiencia de las madres frente a la asistencia a los controles de oxígeno es un hecho que las conmueve profundamente, pues es su primera cita y les da temor saber cómo está él bebe, si se le puede quitar el oxígeno, también manifestaron que para asistir a los controles se requiere de cuidados extremos de los cilindros de oxígeno, del humidificador y de la cánula nasal. Relataron que asistieron a la consulta al día siguiente de la salida del hospital con sus parejas y acompañantes, pero ellos muchas veces tienen que dejar de ir a trabajar para acompañarlas a los controles, así como dejar de hacer algunas actividades de su vida cotidiana. 
Algunas madres entrevistadas indicaron que acudieron al control del oxígeno como fue indicado a antes de la salida del hospital y que han asistido a todos los controles programados de oxígeno. Una de las madres manifestó que: «no ha asistido a todos los controles de oxígeno porque tiene otros niños pequeños y no tiene quién se los cuide».

\section{Discusiones}

Conocer las vivencias de las madres de bebés prematuros con oxígeno es importante para identificar las necesidades y facilitar el proceso de adaptación en su hogar. Las madres expresaron diferentes sentimientos relacionados con tener "un hijo canguro" algunas manifestaron que ha sido una gran experiencia, aunque relatan que es una lucha diaria, ellas mismo se cuestionan sobre si los cuidados que tienen con su hijo son los mejores.

Como describe Castiblanco López (10), su estudio motivó la decisión de construir un recurso pedagógico que ayudara a los padres a cuidar a sus hijos en el hogar cuando estos continuaban con oxígeno. En el estudio se describe el significado que tiene para los padres y sus familias participar en el cuidado directo de su hijo, así como se promulgan recomendaciones encaminadas a fortalecer la educación frente al manejo del oxígeno, ya que detectaron que los padres presentaban dificultades relacionadas con los equipos de oxígeno.

Así como se describen en otras investigaciones de vivencias de padres de bebes prematuros, las madres manifestaron sentimientos de incapacidad para cuidar a su hijo en el momento de ser dados de alta del hospital (11), relataron lo difícil que es tener un bebe canguro con oxígeno porque es estar todo el día en el pecho con él, impidiendo hacer más actividades en casa.

El conocimiento de los padres sobre la prematuridad no se aleja de las definiciones encontradas en diversos artículos de neonatología. Se define prematuro a un bebe nacido vivo antes de que se hayan cumplido 37 semanas de gestación (12).

La prematuridad es considerada un problema de salud pública, con una elevada mortalidad y con altos costos sociales y económicos ocasionados por la hospitalización. La prevención de la prematuridad es una política pública nacional e involucra a muchos actores, se puede evitar el parto prematuro si se identifican de forma temprana los factores predisponentes. Las estrategias empleadas para prevenir y tratar al parto prematuro son amplias y van desde los cuidados preconcepcionales, hasta la atención del parto y del neonato en el periodo postnatal (13).

Según Charpak y cols. (14), el seguimiento del bebe prematuro debe ir mucho más allá del primer año de edad corregida, en Colombia el PMC se realiza hasta los dos años de edad corregida. Hay evidencias que indican que el niño que fue prematuro extremo con bajo peso al nacer tiene más riesgo de presentar alteraciones cognitivas, trastornos del aprendizaje y desadaptación social, estos problemas son más evidentes antes de los dos años de vida, requieren de un seguimiento de alto riesgo y de una intervención terapéutica individual (14).

\section{Conclusiones}

La investigación describe el significado que tienen para las madres los cuidados de su bebe prematuro con oxígeno en casa, este momento generó en ellas estrés, miedo e inseguridad, relataron lo difícil que fue la primera noche en casa y lo importante que es tener confianza y hacer «las cosas bien».

El fortalecimiento de la educación a los padres durante la hospitalización sobre los cuidados del prematuro en casa es fundamental, los relatos de las madres permiten evidenciar que el cuidado de sus hijos requiere de acompañamiento por parte de sus parejas. Comprender las vivencias de las madres no es fácil, pero deja una gran enseñanza para los profesionales de salud y es que el proceso de adaptación de los padres se logra con una comunicación asertiva, con una atención humanizada y permitiéndoles participar en el cuidado de su bebe durante la hospitalización, fortaleciendo en ellos la confianza y seguridad para cuidarlos en casa.

Se debe garantizar a los padres la accesibilidad a los servicios de salud, con el fin de dar continuidad a los procesos de seguimiento que requieren los niños prematuros en los Programas Madre Canguro Ambulatorio, la investigación permite evidenciar que el Programa Ruta de la Salud que les ofrecen a los padres no es suficiente y requiere de una mayor cobertura.

Se debe generar una estrategia que permita realizar un seguimiento y acompañamiento a través de la teleorientación, con el fin de abordar inquietudes que puedan surgir en casa y disminuir en ellos los niveles de ansiedad y estrés. Los resultados de la presente investigación se constituyen en un aporte al talento humano en salud que intervienen en el cuidado del bebe prematuro, a la academia y a los grupos de investigación.

\section{R E F E R E N C I A S}

1. Ministerio de salud y protección social. ImplementacionPrograma-Canguro1. 2017; [Acceso en marzo 6 de 2020]. Disponible en: https://minsalud.gov.co/sites/rid/Lists/BibliotecaDigital/ RIDE/DE/implementacion- programa-canguro.pdf

2. Davidson L, Berkelhamer S. Bronchopulmonary Dysplasia: Chronic Lung Disease of Infancy and Long-Term Pulmonary Outcomes. J Clin Med. 2017;6(1):4.

3. Latini G, De Felice C, Giannuzzi R, Del Vecchio A. Survival rate and prevalence of bronchopulmonary dysplasia in extremely low birth weight infants. Early Hum Dev [2013;89(SUPPL.1): S69-73.

4. Martin RJ, Fanaroff AA. The preterm lung and airway: Past, present, and future. Pediatr Neonatol 2013;54(4):228-34.

5. Seguimiento del recién nacido prematuro y del niño de alto riesgo biológico. [Accesado en marzo 20 de 202]. Disponible en: https://www.pediatriaintegral.es/publicacion-2014-07/seguimiento-delrecien-nacido-prematuro-y-del-nino-de-alto-riesgo-biologico/

6. Álvarez CV. El cuidado en enfermería, perspectiva fenomenológica. Rev Hacia la Promoción la Salud. 2011;16(2):175-90.

7. Giddens A, Bauman Z, Luhmann N, Beck U. Las consecuencias perversas de la modernidad. Recensions. 1998;54:215-9. 
8. Lincoln YS, Guba EG. Ethics: The Failure of Positivist Science. Vol. 12, The Review of Higher Education. 1989. p. 221-40.

9. Manzini JL. Declaración De Helsinki: Principios Éticos Para La Investigación Médica Sobre Sujetos Humanos. Acta Bioeth. 2000;6(2):321-34.

10. Castiblanco López N, Muñoz de Rodríguez L. Visión de las madres en el cuidado del hijo prematuro en el hogar. Av en Enferm. 2011;29(1):120-9.

11. Ocampo MP. El hijo ajeno: Vivencia de madres de niños prematuros hospitalizados. Aquichan. 2013;13(1):69-80.

12. Nacimientos prematuros. [Acceso en marzo 6 de 2020]. Disponible en: 1. https://www.who.int/es/news-room/fact-sheets/detail/ preterm-birth.

13. Mendoza Tascón LA, Claros Benítez DI, Mendoza Tascón LI, Arias Guatibonza MD, Peñaranda Ospina CB. Epidemiología la prematuridad, sus determinantes y prevención del parto prematuro. Rev Chil Obstet Ginecol 2016; 81(4):330-42.

14. Ruiz, J Charpak N. Guías De Práctica Clínica Basadas En Evidencia Para La Óptima Utilización Del Método Madre Canguro De Cuidados Del Recién Nacido Pretérmino y/o De Bajo Peso Al Nacer. 2012; 7-147. 